\title{
Uniform Sampling of the Infinite Noncooperative Game on Unit Hypercube and Reshaping Ultimately Multidimensional Matrices of Player's Payoff Values
}

\author{
Vadim Romanuke (Researcher, Khmelnitskiy National University)
}

\begin{abstract}
The paper suggests a method of obtaining an approximate solution of the infinite noncooperative game on the unit hypercube. The method is based on sampling uniformly the players' payoff functions with the constant step along each of the hypercube dimensions. The author states the conditions for a sufficiently accurate sampling and suggests the method of reshaping the multidimensional matrix of the player's payoff values, being the former player's payoff function before its sampling, into a matrix with minimally possible number of dimensions, where also maintenance of one-to-one indexing has been provided. Requirements for finite NE-strategy from NE (Nash equilibrium) solution of the finite game as the initial infinite game approximation are given as definitions of the approximate solution consistency. The approximate solution consistency ensures its relative independence upon the sampling step within its minimal neighborhood or the minimally decreased sampling step. The ultimate reshaping of multidimensional matrices of players' payoff values to the minimal number of dimensions, being equal to the number of players, stimulates shortened computations.
\end{abstract}

Keywords - Systems, man, and cybernetics; Decision theory; Computational efficiency; Mathematical model.

\section{NONCOOPERATIVE GAME MODELS}

Mathematically, a game is a means for rational resources allocation to meet the growing demands and requirements. And many events, systems and processes, related to the inequality between resources and pretensions, are modeled with noncooperative games [1], [2]. Particularly, these games involve a few players in military process and jurisprudence [3], [4]. Also, there may be more players (up to a few tens) in economical and social-ecological gaming models [5], [6]. Sometimes a two-sided noncooperative game is the most appropriate model for removing uncertainties in technical problems [6], [7]. For instance, this is for preventing a denial of service, when a server (reservoir) runs out of resources while a number of queries (demands) is not less than the rejection number [8], [9].

Naturally, that far from any noncooperative game solution in NE (Nash equilibrium) strategies ensures equilibrium, utility, and fairness [2], [10], [11]. However, NE-solutions render a lot of the refined or modified principles of optimality, allowing to smooth differences in utility and equity [2], [10], [11]. Mainly, they are principles of Pareto equilibrium [2], [6], [8], [10], [13], [14], Mertens-stable equilibrium [15], trembling hand perfect equilibrium [16], proper equilibrium [17], [18], correlated equilibrium [19], sequential equilibrium [20], [21], quasi-perfect equilibrium [18], [22], [23], perfect
Bayesian equilibrium [18], [20], [24], [25], quantal response equilibrium [26], [27], self-confirming equilibrium [28], [29], strong Nash equilibrium [30], [31], Markov perfect equilibrium [32], [33]. The question is only to find NEsolutions as fast as possible.

\section{SOLVING NONCOOPERATIVE GAMES ON COMPACT ACTION SPACES}

Finding NE-solutions in even the finite noncooperative game is a computational difficulty [8], [34], [35]. Locally, solving dyadic games with three players takes some technique of visualization of the cube of situations in pure strategies [6], [10], whereupon dyadic games with four players and more are solved purely in analytics, requiring more computational resources [10], [36]. Naturally, that finite noncooperative games with greater numbers of pure strategies at their players (three and more) are significantly hard to solve them [10], [37], [38]. Moreover, often an admissible player's action is described with a series of its continuous parameters, constituting thus an infinite (continuous) set of pure strategies [1], [6], [7], [12], [39], [40]. If this continuous set is compact then it is easy to find an isomorphic game to the initial one, that the set of every player's pure strategies would be Euclidean finite-dimensional subspace [6], [10], [12], [23], [41]. Normally, the spoken subspace may be a unit cube of the appropriate dimension [6], [10], [11]. Nonetheless, compact games, having solutions at least in mixed strategies for measurable payoff functions [1], [6], [10], [11], [41], [42], cannot be solved by a universal algorithmic approach, unless they are finite games.

\section{TASKS FOR THE GOAL ATTAINMENT}

Clearly, a proper conversion of the infinite noncooperative game on unit hypercube into a finite game lets to have a guaranteed NE-solution [6], [10] to the conflict object. Therefore, the goal of this paper is to state the conditions or requirements of that conversion. To simplify the finite game more there will be reconfigured sets of players' pure strategies, letting to get rid of dimensionalities and to have the single dimension for each player. For the goal attainment there are tasks to state the following:

1. Conditions for sampling the players' payoff functions correctly, being sufficiently accurate for practice experience.

2. Method of reshaping the multidimensional matrix of the player's payoff values (being the former player's payoff function before its sampling) into a matrix with minimally 
possible number of dimensions. Maintenance of one-to-one indexing should be provided.

3. Requirements on that any finite NE-strategy (from NEsolution of the finite game as the approximation of the initial infinite game) must not be too dependent upon the sampling step. Or, in other words, NE-strategy support must be independent upon the sampling step within some tolerable dependence.

\section{SAMPLING OF PLAYERS’ PAYOFF FUNCTIONS}

Consider a noncooperative game

$$
\left\langle\left\{U_{n}\right\}_{n=1}^{N},\left\{K_{n}(\mathbf{X})\right\}_{n=1}^{N}\right\rangle
$$

of $N \in \mathbb{N} \backslash\{1\}$ players, in which the $n$th player acts within the unit hypercube $U_{n}$ of $M_{n}$-dimensional points (pure strategies)

$$
\begin{gathered}
\mathbf{X}_{n}=\left[x_{n m}\right]_{1 \times M_{n}} \in \bigcup_{m=1}^{M_{n}}[0 ; 1]=U_{n} \subset \mathbb{R}^{M_{n}} \\
\text { by } M_{n} \in \mathbb{N} \quad \forall n=\overline{1, N},
\end{gathered}
$$

and in the situation

$$
\mathbf{X}=\left\{\mathbf{X}_{n}\right\}_{n=1}^{N} \in \chi_{n=1}^{N} U_{n}=\sum_{k=1}^{\sum_{i=1}^{N} M_{i}}[0 ; 1] \subset \mathbb{R}^{\sum_{i=1}^{N} M_{i}}
$$

gets the payoff $K_{n}(\mathbf{X})$. Each of the measurable payoff functions $\left\{K_{n}(\mathbf{X})\right\}_{n=1}^{N}$ is defined on the unit $\left(\sum_{i=1}^{N} M_{i}\right)-$ dimensional hypercube

$$
U=\chi_{n=1}^{N} U_{n}=\chi_{k=1}^{\sum_{i=1}^{N} M_{i}}[0 ; 1] \subset \mathbb{R}^{\sum_{i=1}^{N} M_{i}}
$$

and it is supposed that they all are differentiable with respect to any of variables $\left\{\left\{x_{n m}\right\}_{m=1}^{M}\right\}_{n=1}^{N}$. Also there exist mixed derivatives of each of functions $\left\{K_{n}(\mathbf{X})\right\}_{n=1}^{N}$ by any combination of variables $\left\{\left\{x_{n m}\right\}_{m=1}^{M}\right\}_{n=1}^{N}$ in any situation (3), where every variable is included no more than just once.

While sampling uniformly, let $S$ be the number of intervals between the selected points in each of the dimensions of every hypercube from $\left\{U_{n}\right\}_{n=1}^{N}$. Remember that each dimension is the unit segment now. Tolerating the utmost case of sampling, where endpoints of the unit segment are included into the sampling necessarily, $S \in \mathbb{N}$. The constant sampling step is $S^{-1}$. Thus in $m$ th dimension $n$th player instead of the segment $[0 ; 1]$ of values of $m$ th component of its pure strategy $\mathbf{X}_{n}$ in (2) now possesses the set of points

$$
\begin{gathered}
D_{m}^{\langle n\rangle}(S)=\left\{x_{n m}^{\langle s\rangle}\right\}_{s=1}^{S+1} \\
\text { by } x_{n m}^{\langle s\rangle}=\frac{s-1}{S} \quad \forall m=\overline{1, M_{n}} \text { and } \forall n=\overline{1, N} .
\end{gathered}
$$

The choice of number $S$ should not annul specificities of the players' payoff functions. These specificities consist in local extremums and gradient over hypersurfaces $\left\{K_{n}(\mathbf{X})\right\}_{n=1}^{N}$. That is $\forall s=\overline{1, S}$ there ought to be

$$
\begin{gathered}
\frac{\partial^{\sum_{i=1}^{N} M_{i}} K_{n}(\mathbf{X})}{\partial x_{11} \partial x_{12} \ldots \partial x_{1 M_{1}} \partial x_{21} \partial x_{22} \ldots \partial x_{2 M_{2}} \ldots \partial x_{N 1} \partial x_{N 2} \ldots \partial x_{N M_{N}}} \geqslant 0 \text { or } \\
\frac{\partial^{\sum_{i=1}^{N} M_{i}} K_{n}(\mathbf{X})}{\partial x_{11} \partial x_{12} \ldots \partial x_{1 M_{1}} \partial x_{21} \partial x_{22} \ldots \partial x_{2 M_{2}} \ldots \partial x_{N 1} \partial x_{N 2} \ldots \partial x_{N M_{N}}} \leqslant 0 \\
\forall x_{n m} \in\left[x_{n m}^{\langle s\rangle} ; x_{n m}^{\langle s+1\rangle}\right], \forall m=\overline{1, M_{n}}, \forall n=\overline{1, N},
\end{gathered}
$$

and

$$
\begin{aligned}
& \left|\frac{\partial^{\sum_{i=1}^{N} M_{i}} K_{n}(\mathbf{X})}{\partial x_{11} \partial x_{12} \ldots \partial x_{1 M_{1}} \partial x_{21} \partial x_{22} \ldots \partial x_{2 M_{2}} \ldots \partial x_{N 1} \partial x_{N 2} \ldots \partial x_{N M_{N}}}\right| \leqslant \alpha \\
& \forall x_{n m} \in\left[x_{n m}^{\langle s\rangle} ; x_{n m}^{\langle s+1\rangle}\right], \forall m=\overline{1, M_{n}}, \forall n=\overline{1, N}
\end{aligned}
$$

for some $\alpha>0$, implying tolerable fluctuations of the players' payoff functions on every of segments

$$
\left\{\left\{\left\{\left[x_{n m}^{\langle s\rangle} ; x_{n m}^{\langle s+1\rangle}\right]\right\}_{s=1}^{S}\right\}_{m=1}^{M_{n}}\right\}_{n=1}^{N} .
$$

But here it is clear that conditions (6) are satisfied only if there are no extremums on any of intervals

$$
\left\{\left\{\left\{\left(x_{n m}^{\langle s\rangle} ; x_{n m}^{\langle s+1\rangle}\right)\right\}_{s=1}^{S}\right\}_{m=1}^{M_{n}}\right\}_{n=1}^{N} .
$$

Obviously, the players' payoff functions, whose extremums can be reached at points, having only components $\left\{\left\{\left\{x_{n m}^{\langle s\rangle}\right\}_{s=2}^{S}\right\}_{m=1}^{M_{n}}\right\}_{m=1}^{N}$, can be defined just artificially, rather than be expected to occur for real conflict processes. Hence, the real conditions for sampling the players' payoff functions correctly remain in inequalities (7). The conditions (6) may be optionally checked to underscore the accurateness of the sampling (but they will be unlikely satisfied, unless there are two players and minimum of dimensions).

Parameter $\alpha$ is pre-assigned on some practical reasoning. Considering the value

$$
v_{\alpha}=\max _{n \in\{1, N\}} \max _{\mathbf{X} \in U} K_{n}(\mathbf{X})-\min _{n \in\{1, N\}} \min _{\mathbf{X} \in U} K_{n}(\mathbf{X}),
$$

it can be said that $\alpha \leqslant 0.01 \cdot v_{\alpha}$ or $\alpha \leqslant 0.001 \cdot v_{\alpha}$, are sufficiently accurate for practice experience. Nevertheless, the parameter $\alpha$ may be taken lesser to have the game 
approximate solution consistent enough, what is going to be spoken about further.

\section{RESHAPING OF MULTIDIMENSIONAL MATRICES OF PLAYERS' PAYOFF VALUES}

After having sampled the hypersurfaces $\left\{K_{n}(\mathbf{X})\right\}_{n=1}^{N}$ on the unit hypercube (4) the $n$th player gets $\left(\sum_{i=1}^{N} M_{i}\right)$-dimensional matrix $\mathbf{P}_{n}=\left[p_{J}^{\langle n\rangle}\right]_{\mathbf{F}}$ of the format $\mathbf{F}=\sum_{k=1}^{\sum_{i=1}^{N} M_{i}}(S+1)$, whose $\left(\sum_{i=1}^{N} M_{i}\right)$-position indices

$$
J=\left\{j_{k}\right\}_{k=1}^{\sum_{i=1}^{N} M_{i}} \quad \text { by } \quad j_{k} \in\{\overline{1, S+1}\} \quad \forall k=\overline{1, \overline{\sum_{i=1}^{N} M_{i}}}
$$

determine the matrix element

$$
\begin{gathered}
p_{J}^{\langle n\rangle}=K_{n}(\mathbf{X}) \text { by } x_{r m}=\frac{j_{k}-1}{S} \\
\text { at } k=m+\sum_{i=1}^{r-1} M_{i} \quad \forall m=\overline{1, M_{r}} \text { and } \forall r=\overline{1, N} .
\end{gathered}
$$

Understandably, it is very inconvenient to operate on such multidimensional matrices, representing the players' payoff values. Another inconvenience is computational retardation due to supplementary dimensions. For instance, 1000 operations of summing and extracting mean that minimal and maximal elements of $50 \times 50 \times 50 \times 50$-matrix in Matlab take about 303 seconds, whereas the same takes about 295 seconds over the matrix reshaped into $2500 \times 2500$-matrix. Therefore matrices $\left\{\mathbf{P}_{n}\right\}_{n=1}^{N}$ should be reshaped to reduce the number of their dimensions ultimately. The minimal number of dimensions, apparently, is the number of players.

Before getting started, note that the subset of indices $\left\{j_{k}\right\}_{k=1+\sum_{i=1}^{n} M_{i}^{n} M_{i}} \subset J$ corresponds to a pure strategy of the $n$th player, $n=\overline{1, N}$. Then $\mathbf{F}$-matrix $\mathbf{P}_{n}=\left[p_{J}^{\langle n\rangle}\right]_{\mathbf{F}}$ is reshaped into $\chi_{r=1}^{N}(S+1)^{M_{r}}$-matrix

$$
\mathbf{G}_{n}=\left[g_{L}^{\langle n\rangle}\right]_{\mathbf{L}}
$$

of elements $g_{L}^{\langle n\rangle}=p_{J}^{\langle n\rangle}$, whose indices within the format $\mathbf{L}=\chi_{r=1}^{N}(S+1)^{M_{r}}$ are in the set

$$
\begin{gathered}
L=\left\{u_{r}\right\}_{r=1}^{N} \text { by } u_{r}=\sum_{m=1}^{M_{r}}(S+1)^{m-1} \cdot\left(j_{k}-\operatorname{sign}(m-1)\right) \\
\text { at } k=\sum_{i=1}^{r} M_{i}-m+1 \text { and } \forall r=\overline{1, N} .
\end{gathered}
$$

Henceforward, instead of the infinite noncooperative game (1) on unit hypercube (4) here is its approximation as the finite noncooperative game

$$
\left\langle\left\{\left\{z_{u_{n}}^{\left\langle\mathbf{X}_{n}\right\rangle}(S)\right\}_{u_{n}=1}^{(S+1)^{M_{n}}}\right\}_{n=1}^{N},\left\{\mathbf{G}_{n}\right\}_{n=1}^{N}\right\rangle
$$

where the $n$th player's pure strategy $z_{u_{n}}^{\left\langle\mathbf{X}_{n}\right\rangle}(S)$ corresponds to its strategy $\mathbf{X}_{n}$ in the initial game (1), whose components are

$$
\left\{x_{n m}=\frac{j_{k}-1}{S}\right\}_{m=1}^{M_{n}} \text { at } k=m+\sum_{i=1}^{n-1} M_{i} \text { by } n=\overline{1, N} \text {. }
$$

For the $n$th player, the transition from the matrix $\mathbf{P}_{n}=\left[p_{J}^{\langle n\rangle}\right]_{\mathrm{F}}$ to the matrix (10), implying the mapping of the indices set (8) into the indices set (11), is reversible. This is maintained by that the subset of indices $\left\{j_{k}\right\}_{k=1+\sum_{i=1}^{n} M_{i}}^{n} \subset J$, corresponding to the pure strategy $z_{u_{n}}^{\left\langle\mathbf{X}_{n}\right\rangle}(S)$ in the game (12), is found by the index $u_{n}$ :

$$
\left.\begin{array}{c}
j_{k}=\eta\left(u_{n}, S+1\right)+(S+1)\left(1-\operatorname{sign}\left[\eta\left(u_{n}, S+1\right)\right]\right) \\
\text { at } k=\sum_{i=1}^{n} M_{i}, \\
j_{k-m}=1+\eta\left(\frac{u_{n}-j_{k}-\sum_{q=1}^{m-1}(S+1)^{q}\left(j_{k-q}-1\right)}{(S+1)^{m}}, S+1\right) \\
\forall m=\overline{1, M_{n}-1}
\end{array}\right)
$$

by the function $\eta(y, b)$ returning the fractional part (remainder after division) of the ratio $\frac{y}{b}$. Then the set (8) is indeed given back, whence the matrix element (9) can be restored.

\section{CONSISTENCY OF NE-STRATEGY SUPPORT, APPROXIMATING THE UNKNOWN GENUINE NE-STRATEGY}

May the set

$$
\left\{\left\{p_{N E}^{\langle n\rangle}\left(z_{u_{n}}^{\left\langle\mathbf{X}_{n}\right\rangle}(S)\right)\right\}_{u_{n}=1}^{(S+1)^{M_{n}}}\right\}_{n=1}^{N}
$$

be the game (12) solution, in which $p_{N E}^{\langle n\rangle}\left(z_{u_{n}}^{\left\langle\mathbf{X}_{n}\right\rangle}(S)\right)$ is the 
probability of applying the pure strategy $z_{u_{n}}^{\left\langle\mathbf{X}_{n}\right\rangle}(S)$ in an NEstrategy of the $n$th player

$$
\left\{p_{N E}^{\langle n\rangle}\left(z_{u_{n}}^{\left\langle\mathbf{X}_{n}\right\rangle}(S)\right)\right\}_{u_{n}=1}^{(S+1)^{M_{n}}}
$$

And may the support of the $n$th player's NE-strategy (16) be the set

$$
\left\{z_{u_{n}^{\langle q\rangle}(S)}^{\left\langle\mathbf{X}_{n}\right\rangle}(S)\right\}_{q=1}^{Q_{n}^{*}(S)}, \quad\left\{u_{n}^{\langle q\rangle}(S)\right\}_{q=1}^{Q_{n}^{*}(S)} \subset\left\{\overline{1,(S+1)^{M_{n}}}\right\},
$$

having its cardinality $Q_{n}^{*}(S) \leqslant(S+1)^{M_{n}}$ by the number $S$ of intervals between the selected points in each of dimensions of hypercube $U_{n}$. Then the $n$th player's NE-strategy (16) has the property:

$$
p_{N E}^{\langle n\rangle}\left(z_{u_{n}}^{\left\langle\mathbf{X}_{n}\right\rangle}(S)\right)>0 \quad \forall u_{n} \in\left\{u_{n}^{\langle q\rangle}(S)\right\}_{q=1}^{Q_{n}^{*}(S)}
$$

and

$$
p_{N E}^{\langle n\rangle}\left(z_{u_{n}}^{\left\langle\mathbf{X}_{n}\right\rangle}(S)\right)=0 \quad \forall u_{n} \notin\left\{u_{n}^{\langle q\rangle}(S)\right\}_{q=1}^{Q_{n}^{*}(S)} .
$$

The task now is to see how badly the approximate solution will change if the number of intervals between the selected points in each of dimensions of hypercubes $\left\{U_{n}\right\}_{n=1}^{N}$ is increased minimally. For that there must be compared not just approximate solutions (15) and

$$
\left\{\left\{p_{N E}^{\langle n\rangle}\left(z_{u_{n}}^{\left\langle\mathbf{X}_{n}\right\rangle}(S+1)\right)\right\}_{u_{n}=1}^{(S+2)^{M_{n}}}\right\}_{n=1}^{N}
$$

but also the players' payoffs. And conception of the Helly metric $[6,10,11]$ couldn't be applied here inasmuch as the couple of each player's NE-strategies from solutions (15) and (20) is compiled from diverse games.

In this connection denote by $v_{N E}^{\langle n\rangle}(S)$ the $n$th player's payoff, being taken in the factual NE-situation (15):

$$
\begin{gathered}
v_{N E}^{\langle n\rangle}(S)=\sum_{\substack{L=\left\{u_{l}\right\}_{l=1}^{N} \\
u_{l}=1,(S+1)^{M_{l}}}}\left(g_{L}^{\langle n\rangle} \cdot \prod_{i=1}^{N} p_{N E}^{\langle i\rangle}\left(z_{u_{i}}^{\left\langle\mathbf{X}_{i}\right\rangle}(S)\right)=\right. \\
=\sum_{\substack{L_{*}(S)=\left\{u_{l}^{\langle q\rangle}(S)\right\}_{l=1}^{N} \\
q=1, Q_{n}^{*}(S)}}\left(g_{L_{t}(S)}^{\langle n\rangle} \cdot \prod_{i=1}^{N} p_{N E}^{\langle i\rangle}\left(z_{u_{i}^{\langle q\rangle}(S)}^{\left\langle\mathbf{x}_{i}\right\rangle}(S)\right)\right), n=\overline{1, N} .
\end{gathered}
$$

So, $N$ players take their payoffs $\left\{v_{N E}^{\langle n\rangle}(S)\right\}_{n=1}^{N}$ in NEsituation (15), and they take payoffs $\left\{v_{N E}^{\langle n\rangle}(S+1)\right\}_{n=1}^{N}$ in NEsituation (20) for the minimally increased sampling number (the minimally decreased sampling step). Apparently, there can be selected such a sampling step, for which at least $\exists n_{0} \in\{\overline{1, N}\}$ such that payoffs $v_{N E}^{\left\langle n_{0}\right\rangle}(S)$ and $v_{N E}^{\left\langle n_{0}\right\rangle}(S+1)$ will be significantly different. Another difference is that NE- situation (15) in the game (12) will have configuration, being hardly comparable to the corresponding configuration of NEsituation in the game (1) or to NE-situation (20) for the minimally decreased sampling step. So, for initial acceptance of the game (12) solution as an approximate solution of the game (1) there are the following exigencies:

1) a sufficient closeness of the players' NE-situations, being found by nearest neighbor numbers of intervals between the selected points in each of dimensions of every hypercube from $\left\{U_{n}\right\}_{n=1}^{N}$

2) a sufficient closeness of the players' payoffs, being taken in these NE-situations.

However, what is the rate of "sufficient closeness" for those ones? Obviously, it is unknown as well as the players' payoffs are. The one that remains there is a relative closeness, meaning that the attribute value (the player's payoff, the player's NE-strategy, etc.) differentiates less as the number $S$ increases (growing more "stable"). In the payoff case, that relative closeness of the players' payoffs is this

$$
\begin{gathered}
\left|v_{N E}^{\langle n\rangle}(S)-v_{N E}^{\langle n\rangle}(S+1)\right| \leqslant\left|v_{N E}^{\langle n\rangle}(S-1)-v_{N E}^{\langle n\rangle}(S)\right| \\
\forall n=\overline{1, N} .
\end{gathered}
$$

The sufficient closeness in the case of NE-situations, giving payoffs for players, needs consideration of the player's NEstrategies supports as hypersurfaces. Let for the $n$th player there be a piecewise linear hypersurface $h_{n}\left(u_{n}, S\right)$, vertices of which are in points

$$
\begin{gathered}
\left\{\left\{\left[\frac{j_{k}-1}{S}\right]_{1 \times M_{n}} \in \mathbb{R}^{M_{n}}: k=m+\sum_{i=1}^{n-1} M_{i}, m=\overline{1, M_{n}}\right\},\right. \\
\left.p_{N E}^{\langle n\rangle}\left(z_{u_{n}}^{\left\langle\mathbf{X}_{n}\right\rangle}(S)\right)\right\}
\end{gathered}
$$

in the space $\mathbb{R}^{M_{n}+1}$. The $n$th player's NE-strategy support (17) scores up the nonzero vertices of the hypersurface $h_{n}\left(u_{n}, S\right)$ by (18) and (19), wherein

$$
\begin{gathered}
\mathbf{X}_{n}^{\langle q\rangle}(S)=\left[x_{n m}^{\langle q\rangle}(S)\right]_{1 \times M_{n}}=\left[\frac{j_{k}^{\langle q\rangle}(S)-1}{S}\right]_{1 \times M_{n}} \in U_{n} \\
\text { by } k=m+\sum_{i=1}^{n-1} M_{i} \text { and } m=\overline{1, M_{n}}
\end{gathered}
$$

by (13) for $q=\overline{1, Q_{n}^{*}(S)}$ and matching the index $u_{n}^{\langle q\rangle}(S)$ to the point (24) through the expansion (14). Then may the $n$th player's set $\left\{\mathbf{X}_{n}^{\langle q\rangle}(S)\right\}_{q=1}^{Q_{n}^{*}(S)}$ of the points (24) be sorted into the set

$$
\left\{\overline{\mathbf{X}}_{n}^{\langle q\rangle}(S)\right\}_{q=1}^{Q_{n}^{*}(S)}=\left\{\left[\frac{\bar{j}_{k}^{\langle q\rangle}(S)-1}{S}\right]_{1 \times M_{n}}\right\}_{q=1}^{Q_{n}^{*}(S)}=\left\{\mathbf{X}_{n}^{\langle q\rangle}(S)\right\}_{q=1}^{Q_{n}^{*}(S)}
$$




$$
\text { by } k=m+\sum_{i=1}^{n-1} M_{i} \text { and } m=\overline{1, M_{n}}
$$

so that the value

$$
q_{1} \in\left\{\frac{\min }{q+1, Q_{n}^{*}(S)}\right\} \sqrt{\sum_{k=m+\sum_{i=1}^{n-1} M_{i}, m=1, M_{n}}\left(\bar{j}_{k}^{\langle q\rangle}(S)-\bar{j}_{k}^{\left\langle q_{1}\right\rangle}(S)\right)^{2}}
$$

is reached at $q_{1}=q+1$ for each $q=\overline{1, Q_{n}^{*}(S)-1} \forall n=\overline{1, N}$. With new indices $\left\{\bar{j}_{k}^{\langle q\rangle}(S)\right\}_{q=1}^{Q_{n}^{*}(S)}$ of the sets (25) $\forall n=\overline{1, N}$ there appears an enhancement in measuring the averaged support density. This is needed because the inequality

$$
Q_{n}^{*}(S+1) \geqslant Q_{n}^{*}(S) \quad \forall n=\overline{1, N}
$$

cannot singly itself express the requirement of that the averaged support density shall not decrease for the minimally decreased sampling step (the minimally increased sampling number).

Definition 1. The solution (15) of the game (12) is called weakly consistent for being the approximate solution of the game (1) if $\forall n=\overline{1, N}$ the inequalities

$$
\begin{gathered}
\sum_{q \in\left\{\frac{\max }{1, Q_{n}^{*}(S+1)-1}\right\}} \sqrt{\sum_{k=m+\sum_{i=1}^{n-1} M_{i}, m=\overline{1, M_{n}}}\left(\bar{j}_{k}^{\langle q\rangle}(S+1)-\bar{j}_{k}^{\langle q+1\rangle}(S+1)\right)^{2}} \leqslant \\
\leqslant \sum_{q \in\left\{\frac{\max }{1, Q_{n}^{*}(S)-1}\right\}} \sqrt{\sum_{k=m+\sum_{i=1}^{n-1} M_{i}, m=\overline{1, M_{n}}}\left(\bar{j}_{k}^{\langle q\rangle}(S)-\bar{j}_{k}^{\langle q+1\rangle}(S)\right)^{2}}, \\
\max _{U_{n}}\left|h_{n}\left(u_{n}, S\right)-h_{n}\left(u_{n}, S+1\right)\right| \leqslant \\
\leqslant \max _{U_{n}}\left|h_{n}\left(u_{n}, S-1\right)-h_{n}\left(u_{n}, S\right)\right|
\end{gathered}
$$

and

$$
\begin{gathered}
\left\|h_{n}\left(u_{n}, S\right)-h_{n}\left(u_{n}, S+1\right)\right\| \leqslant \\
\leqslant\left\|h_{n}\left(u_{n}, S-1\right)-h_{n}\left(u_{n}, S\right)\right\| \text { in } \mathbb{L}_{2}\left(U_{n}\right)
\end{gathered}
$$

are true along with (22) and (27).

Noting that inequalities (27) and (28) might be stated for the minimally increased sampling step, there is a way to condition the approximate solution harder. This, nonetheless, will underscore the "monotonicity" of NE-strategies in (15) within minimal neighborhood of the sampling step.

Definition 2. The weakly consistent solution (15) of the game (12) is called consistent for being the approximate solution of the game (1) if $\forall n=\overline{1, N}$ the inequalities

$$
Q_{n}^{*}(S) \geqslant Q_{n}^{*}(S-1)
$$

and

$$
q \in\left\{\frac{\max }{1, Q_{n}^{*}(S)-1}\right\} \sqrt{\sum_{k=m+\sum_{i=1}^{n-1} M_{i}, m=\overline{1, M_{n}}}\left(\bar{j}_{k}^{\langle q\rangle}(S)-\bar{j}_{k}^{\langle q+1\rangle}(S)\right)^{2}} \leqslant
$$

$$
\leqslant \max _{q \in\left\{\frac{1, Q_{n}^{*}(S-1)-1}{1}\right\}} \sum_{\sum_{k=m+\sum_{i=1}^{n-1} M_{i}, m=\overline{1, M_{n}}}\left(\bar{j}_{k}^{\langle q\rangle}(S-1)-\bar{j}_{k}^{\langle q+1\rangle}(S-1)\right)^{2}}
$$

are true.

Surely, (weak) consistency of an NE-strategy support, approximating the unknown genuine NE-strategy, is not sufficient to say that the solution (15) is (weakly) consistent. Speaking strictly, for now there is no proof that the consistent NE-strategy support causes at least the weak consistency of the other NE-strategy support. That is for admission of the solution (15) as an approximate solution of the game (1) there are $5 N$ inequalities (22) and (27)-(30) to be checked. And if one wants to handle the game (1) approximation surer there are $7 N$ inequalities (22) and (27)-(32) to be checked.

Properly speaking, neither conditions within Definition 1, nor conditions within Definition 2 guarantee the perfection of the game (1) approximation as the game (12) with its (weakly) consistent solution (15). But with (22), (29), (30) there is the solution (15) distinctive property signifying that both the players' payoffs (21) and the players' NE-strategies supports as hypersurfaces differentiate less as the number $S$ increases. Growing more "stable", the volume and the averaged NEstrategies supports' densities also do not decrease as the number $S$ increases due to (27) and (28). This "nondecreasing" property becomes stronger with (31)-(27) and (32)-(28), strengthening the solution (15) relative independence upon the sampling step within its minimal neighborhood.

\section{DISCUSSION AND CONCLUSIVE REMARKS}

The conception of consistency has been contrived for proper approximation of the infinite noncooperative game. Being defined on the unit hypercube (4), this game is isomorphic to games, defined on compact subspaces in $\mathbb{R}^{\sum_{n=1}^{N} M_{n}}$, wherein the $n$th player acts within the compact subspace of $\mathbb{R}^{M_{n}}$ by $n=\overline{1, N}$. According to the isomorphism, for solving infinite noncooperative games on compact action spaces there is available the stated approximation way to be applied, allowing to reshape multidimensional matrices of players' payoff values by (11) to the minimal number of dimensions, being equal to the number of players. Due to narrowing the dimensionality, this anyhow shortens the computation period. And computing the factual solution stays for finite noncooperative game solvers [1, 10, 38, 43, 44].

Before approximating, the weak consistency ought to be checked first. The check consecution starts with checking the inequalities (27), where two games are solved towards (15) and (20). Then goes subconsecution of checking the inequalities (22), (29) and (30) $\forall n=\overline{1, N}$, needing three games to be solved, towards (15), (20) and

$$
\left\{\left\{p_{N E}^{\langle n\rangle}\left(z_{u_{n}}^{\left\langle\mathbf{X}_{n}\right\rangle}(S-1)\right)\right\}_{u_{n}=1}^{S^{M_{n}}}\right\}_{n=1}^{N}
$$


Finally, the inequalities (28) $\forall n=\overline{1, N}$ are checked, needing more computational resources for sorting sets $\left\{\left\{\mathbf{X}_{n}^{\langle q\rangle}(S)\right\}_{q=1}^{Q_{n}^{*}(S)}\right\}_{n=1}^{N}$ into sets $\left\{\left\{\overline{\mathbf{X}}_{n}^{\langle q\rangle}(S)\right\}_{q=1}^{Q_{n}^{*}(S)}\right\}_{n=1}^{N}$ although using the previously solved two games with (15) and (20). When the weakly consistent solution (15) of the game (12) is checked for consistency, there are used solutions (15) and (33) once again, and then $N$ inequalities (31) are checked first, whereupon come those $N$ inequalities (32). Namely the stated consecutions are preferable, because the easiest requirements are checked before the more complicated ones in order to prevent needless huge computations (manipulations) over nonconsistent solutions, being exposed after easier comparisons like (27) or (22).

The main lack of the approximation is that there is neither proved limits

$$
\lim _{S \rightarrow \infty} v_{N E}^{\langle n\rangle}(S) \quad \forall n=\overline{1, N}
$$

existence and their convergence to the genuine players' payoffs in NE-situation approximated by NE-situation (15), nor proved limits

$$
\lim _{S \rightarrow \infty} h_{n}\left(u_{n}, S\right) \quad \forall n=\overline{1, N}
$$

existence and their convergence to the hypersurfaces from the genuine NE-situation approximated by NE-situation (15). But anyway, the represented method of converting the infinite noncooperative game on unit hypercube into the finite game lets to have an NE-solution to the conflict object, even when the game (1) is solved in $\varepsilon$-equilibrium situations or doesn't have solution at all. Besides, the approximate solution (15), where every player has the finite NE-strategy support, is practiced more freely with discrete variates [45], [46] unlike practicing on continuous variates [47], [48].

The investigation of approximating infinite noncooperative games could be brought forward if the set in (5) for $n$th player was formed with a specific number of intervals $S_{n}$ between the selected points in each of hypercube $U_{n}$ dimensions. Further to this, $n$th player could take a specific number of intervals $S_{n m}$ between the selected points in $m$ th dimension of one's pure strategy (2). This might be useful inasmuch as multidimensional matrices of players' payoff values in the hypercubic lattice form are preferred to other forms [10]. However, the main issue is to make problems of the type (26) effectively computable for accelerating the consistency checks in (28) and (32).

\section{ACKNOWLEDGMENT}

The work is technically supported by the Parallel Computing Center at Khmelnitskiy National University, Ukraine (http://parallelcompute.sourceforge.net).

\section{REFERENCES}

[1] M. J. Osborne, An introduction to game theory. Oxford: Oxford University Press, Incorporated, 2003.

[2] J. C. Harsanyi and R. Selten, A General Theory of Equilibrium Selection in Games. Cambridge: The MIT Press, 1988.

[3] V. G. Suzdal, Game theory for Fleet. Moscow: Voyenizdat, 1976 (in Russian).

[4] R. Calvert, M. D. McCubbins, and B. R. Weingast, "A Theory of Political Control and Agency Discretion," American J. of Political Science, vol. 33, no. 3, 1989, pp. 588-611. http://dx.doi.org/10.2307/2111064

[5] D. Friedman, "On economic applications of evolutionary game theory," J. of Evolutionary Economics, vol. 8, iss. 1, 1998, pp. 15-43. http://dx.doi.org/10.1007/s001910050054

[6] N. N. Vorobyov, Game theory for economist-cyberneticians. Moscow: Nauka, 1985 (in Russian).

[7] V. V. Romanuke, "A theorem on continuum of the projector optimal behaviors in antagonistic model of building resources distribution under segment uncertainties with incorrectly pre-evaluated one left and one right endpoints," Problems of tribology, no. 4, 2011, pp. 52-55.

[8] D. Gasior and M. Drwal, "Pareto-optimal Nash equilibrium in capacity allocation game for self-managed networks," Computer Networks, vol. 57, iss. 14, 2013, pp. 2817-2832. http://dx.doi.org/10.1016/j.comnet.2013.06.012

[9] M. Kucukmehmetoglu, "An integrative case study approach between game theory and Pareto frontier concepts for the transboundary water resources allocations," J. of Hydrology, vol. 450-451, 2012, pp. 308-319.

[10] N. N. Vorobyov, Game theory fundamentals. Noncooperative games. Moscow: Nauka, 1984 (in Russian).

[11] G. Owen, Game theory. Philadelphia - London - Toronto: W. B. Saunders Company, 1968.

[12] G. Stoltz and G. Lugosi, "Learning correlated equilibria in games with compact sets of strategies," Games and Economic Behavior, vol. 59, iss. 1, 2007, pp. 187-208. http://dx.doi.org/10.1016/j.geb.2006.04.007

[13] F. Tao et al., "CLPS-GA: A case library and Pareto solution-based hybrid genetic algorithm for energy-aware cloud service scheduling," Applied Soft Computing, vol. 19, 2014, pp. 264-279. http://dx.doi.org/10.1016/j.asoc.2014.01.036

[14] V. Scalzo, "Pareto efficient Nash equilibria in discontinuous games," Economics Letters, vol. 107, iss. 3, 2010, pp. 364-365. http://dx.doi.org/10.1016/j.econlet.2010.03.010

[15] E. Kohlberg and J.-F. Mertens, "On the Strategic Stability of Equilibria," Econometrica, vol. 54, no. 5, 1986, pp. 1003-1037.

[16] R. Selten, "Reexamination of the perfectness concept for equilibrium points in extensive games," Int. J. of Game Theory, vol. 4, iss. 1, 1975, pp. 25-55. http://dx.doi.org/10.1007/BF01766400

[17] R. B. Myerson, "Refinements of the Nash equilibrium concept," Int. J. of Game Theory, vol. 7, iss. 2, 1978, pp. 73-80. http://dx.doi.org/10.1007/BF01753236

[18] E. van Damme, "A relation between perfect equilibria in extensive form games and proper equilibria in normal form games," Int. J. of Game Theory, vol. 13, iss. 1, 1984, pp. 1-13. http://dx.doi.org/10.1007/BF01769861

[19] R. J. Aumann, "Subjectivity and correlation in randomized strategies," $J$. of Mathematical Economics, vol. 1, iss. 1, 1974, pp. 67-96. http://dx.doi.org/10.1016/0304-4068(74)90037-8

[20] D. Fudenberg and J. Tirole, "Perfect Bayesian equilibrium and sequential equilibrium," J. of Economic Theory, vol. 53, iss. 2, 1991, pp. 236-260. http://dx.doi.org/10.1016/0022-0531(91)90155-W

[21] D. Gerardi and R. B. Myerson, "Sequential equilibria in Bayesian games with communication," Games and Economic Behavior, vol. 60, iss. 1, 2007, pp. 104-134. http://dx.doi.org/10.1016/j.geb.2006.09.006

[22] J.-F. Mertens, "Two examples of strategic equilibrium," Games and Economic Behavior, vol. 8, iss. 2, 1995, pp. 378-388. http://dx.doi.org/10.1016/S0899-8256(05)80007-7

[23] E. Bajoori, J. Flesch, and D. Vermeulen, "Perfect equilibrium in games with compact action spaces," Games and Economic Behavior, vol. 82, 2013, pp. 490-502. http://dx.doi.org/10.1016/j.geb.2013.08.002

[24] P. Battigalli, "Strategic Independence and Perfect Bayesian Equilibria," J. of Economic Theory, vol. 70, iss. 1, 1996, pp. 201-234. http://dx.doi.org/10.1006/jeth.1996.0082

[25] C. C. Moallemi, B. Park, and B. Van Roy, "Strategic execution in the presence of an uninformed arbitrageur," J. of Financial Markets, vol. 15, iss. 4, 2012, pp. 361-391. http://dx.doi.org/10.1016/j.finmar.2011.11.002 
[26] B. W. Rogers, T. R. Palfrey, and C. F. Camerer, "Heterogeneous quantal response equilibrium and cognitive hierarchies," J. of Economic Theory, vol. 144, iss. 4, 2009, pp. 1440-1467. http://dx.doi.org/10.1016/j.jet.2008.11.010

[27] R. Golman, "Quantal response equilibria with heterogeneous agents," $J$. of Economic Theory, vol. 146, iss. 5, 2011, pp. 2013-2028. http://dx.doi.org/10.1016/j.jet.2011.06.007

[28] M. Shimoji, "Outcome-equivalence of self-confirming equilibrium and Nash equilibrium," Games and Economic Behavior, vol. 75, iss. 1, 2012, pp. 441-447. http://dx.doi.org/10.1016/j.geb.2011.09.010

[29] A. Gamba, "Learning and evolution of altruistic preferences in the Centipede Game," J. of Economic Behavior \& Organization, vol. 85, 2013, pp. 112-117. http://dx.doi.org/10.1016/j.jebo.2012.11.009

[30] S.-C. Suh, "An algorithm for verifying double implementability in Nash and strong Nash equilibria," Mathematical Social Sciences, vol. 41, iss. 1, 2001, pp. 103-110. http://dx.doi.org/10.1016/S0165-4896(99)00057-8

[31] G. Tian, "Implementation of balanced linear cost share equilibrium solution in Nash and strong Nash equilibria," J. of Public Economics, vol. 76, iss. 2, 2000, pp. 239-261. http://dx.doi.org/10.1016/S00472727(99)00041-9

[32] S. Castro and A. Brandão, "Existence of a Markov perfect equilibrium in a third market model," Economics Letters, vol. 66, iss. 3, 2000, pp. 297-301. http://dx.doi.org/10.1016/S0165-1765(99)00208-6

[33] H. Haller and R. Lagunoff, "Markov Perfect equilibria in repeated asynchronous choice games," J of Mathematical Economics, vol. 46, iss. 6, 2010, pp. 1103-1114. http://dx.doi.org/10.1016/j.jmateco.2009.09.003

[34] D. Han et al., "An improved two-step method for solving generalized Nash equilibrium problems," European J. of Operational Research, vol. 216, iss. 3, 2012, pp. 613-623. http://dx.doi.org/10.1016/j.ejor.2011.08.008

[35] X. Chen and X. Deng, "Recent development in computational complexity characterization of Nash equilibrium," Computer Science Review, vol. 1, iss. 2, 2007, pp. 88-99. http://dx.doi.org/10.1016/j.cosrev.2007.09.002

[36] L. Browning and A. M. Colman, "Evolution of coordinated alternating reciprocity in repeated dyadic games," J. of Theoretical Biology, vol. 229, iss. 4, 2004, pp. 549-557. http://dx.doi.org/10.1016/j.jtbi.2004.04.032

[37] C. E. Lemke and J. T. Howson, "Equilibrium points of bimatrix games," SIAM Journal on Applied Mathematics, vol. 12, no. 2, 1964, pp. 413-423. http://dx.doi.org/10.1137/0112033
[38] N. Nisan et al., Algorithmic Game Theory. Cambridge: Cambridge University Press, 2007. http://dx.doi.org/10.1017/CBO9780511800481

[39] A. S. Belenky, "A 3-person game on polyhedral sets," Computers \& Mathematics with Applications, vol. 28, iss. 5, 1994, pp. 53-56.

[40] J. H. Dshalalow, "On multivariate antagonistic marked point processes," Mathematical and Computer Modelling, vol. 49, iss. 3-4, 2009, pp. $432-452$.

[41] N. S. Kukushkin, "Nash equilibrium in compact-continuous games with a potential," Int. J. of Game Theory, vol. 40, iss. 2, 2011, pp. 387-392. http://dx.doi.org/10.1007/s00182-010-0261-7

[42] A. Meirowitz, "On the existence of equilibria to Bayesian games with non-finite type and action spaces," Economics Letters, vol. 78, iss. 2, 2003, pp. 213-218. http://dx.doi.org/10.1016/S0165-1765(02)00216-1

[43] H. W. Kuhn, "An algorithm for equilibrium points in bimatrix games," in Proc. Nat. Acad. Sci., vol. 47, 1961, pp. 1657-1662. http://dx.doi.org/10.1073/pnas.47.10.1657

[44] S. C. Kontogiannis, P. N. Panagopoulou, and P. G. Spirakis, "Polynomial algorithms for approximating Nash equilibria of bimatrix games," Theoretical Computer Science, vol. 410, iss. 17, 2009, pp. 1599-1606. http://dx.doi.org/10.1016/j.tcs.2008.12.033

[45] V. V. Romanuke, "The principle of optimality problem in the elementary matrix game with the finite number of plays," Bulletin of Khmelnitskiy National University. Technical Sciences, no. 1, 2007, pp. 226-230.

[46] V. V. Romanuke, "Method of realizing the optimal mixed strategies in the matrix game with the empty set of saddle points in pure strategies with the known number of the game plays," Research bulletin of National Technical University of Ukraine "Kyiv Polytechnic Institute", no. 2, 2009, pp. 45-52 (in Ukrainian).

[47] V. V. Romanuke, "Method of practicing the optimal mixed strategy with innumerable set in its spectrum by unknown number of plays," Measuring and Computing Devices in Technological Processes, no. 2, 2008, pp. 196-203.

[48] V. V. Romanuke, "A program function representation for implementing the method of practicing the optimal mixed strategy with innumerable spectrum by unknown number of plays," Optoelectronic InformationPower Technologies, no. 1, 2010, pp. 28-37.

Vadim Romanuke is a researcher with the Applied Mathematics and Social Informatics Department of Khmelnitskiy National University (Ukraine).

Address: 11 Institutskaya Str., 29016, Khmelnitskiy, Ukraine.

E-mail: romanukevadimv@gmail.com 